\title{
Research on Chinese Language Learning Strategies for University Students
}

\author{
Sun Yonghong ${ }^{1}, \mathrm{Xu}$ Xiangdong ${ }^{2}$ \\ ${ }^{1}$ University of Changchun, Jilin Province, China \\ ${ }^{2}$ Education and Science Institute of Jilin Province, China \\ syh526@hotmail.com, jypjyjs@163.com
}

\begin{abstract}
This paper aims at investigating the Chinese language learning strategies employed by university students. Quantitative research methods are used. The paper presents our findings on the learning strategies of university students who study Chinese as a foreign language. 48 valid questionnaires have been collected. The results show that the university students always use Chinese language learning strategies, employing them at high level and the most commonly used by them kinds of strategies are Social strategies, Metacognitive strategies and Cognitive strategies. They sometimes use Compensation strategies, Memory strategies and Affective strategies and their use frequencies are at medium level.

Index Terms - University students, Chinese language, Chinese language learning strategies.
\end{abstract}

\section{I . Introduction}

Since the beginning of language teaching, the ways of improving its quality have been the main, most important goal in study field. The ultimate goal of any kind of language teaching is development of learners and their lifelong learning. As a method of solving problems and achieving goals, studies on learners' learning have appealed to researchers. Many researchers pay close attention to learning strategies. ${ }^{1}$

\section{II . Questions of the study}

This study aims at investigating the Chinese language learning strategies employed by university students. For this purpose, the research questions would be: 1 . What is the general condition of the Chinese language learning strategies that are used by university students who study Chinese as a foreign language? 2. What kinds of Chinese language learning strategies do university students commonly use?

\section{III . Methodology}

\section{A. Instruments}

The Investigation instruments in the research included a questionnaire and SPSS. The questionnaire consists of three parts. The first part is instruction. The second part is SILL ${ }^{2}$ which was revised for foreign language learners. The Likertstyle scale was used to research which language learning strategies participants employed. The third part is a background used to know the participants information such as gender, age, mother tongue, total duration of learning Chinese and the reasons to learn this language. Every participant's scores were determined by summing up the points of each statement.

\section{B. Participants}

The participants recruited for this study were students from Sofia University which provides Chinese language education programs. There are altogether 48 university students' valid answers to the questionnaire. There were 11 male students and 37 female students who ranged in Chinese proficiency from beginner to advanced level and in age from 18 to 29 years.

\section{Procedures}

The surveys were conducted in the participants' classrooms or through e-mail. All the questionnaires were checked one by one and the data were analyzed by SPSS.

\section{IV . Results}

A. What is the general condition of the Chinese learning strategies that are used by university students who study Chinese as a foreign language?

The score of the general condition of the learning strategies used by university students has been calculated to be 3.5.

According to Profile of Results of Oxford (Oxford 1990), the scores averaging from 4.5 to 5.0 are regarded as high, which means that the participants always or almost always use the learning strategies. The scores ranging from 3.5 to 4.4 are also labeled as high, which means that these strategies are usually used. The scores averaging from 2.5 to 3.4 are regarded as medium, which means that the participants sometimes use the learning strategies. The scores ranging from 1.5 to 2.4 are considered low, which means that the participants generally do not use the learning strategies. The scores averaging from 1.0 to 1.4 are also labeled as low, which means that the learning strategies are never or almost never used.

As a result, university students usually use the Chinese learning strategies at high level.

B. What kinds of Chinese language learning strategies do they commonly use?

There are six strategy groups of Chinese language learning strategies. The results show that Social strategies have the highest mean of 4.1 (SD = 0.64). The strategies of the second highest mean are Metacognitive strategies, whose mean is 3.7 $(\mathrm{SD}=0.79)$. The mean of Cognitive strategies is $3.6(\mathrm{SD}=$ 
0.55), thus they rank third. Compensation strategies rank fourth, whose mean is $3.4(\mathrm{SD}=0.63)$. The means of the other two strategies are the same: both Memory strategies $(\mathrm{M}=3.1, \mathrm{SD}=0.43)$ and Affective strategies $(\mathrm{M}=3.1, \mathrm{SD}=0.63)$ have mean of 3.1. The scores of the six strategy groups are shown in Figure 1.

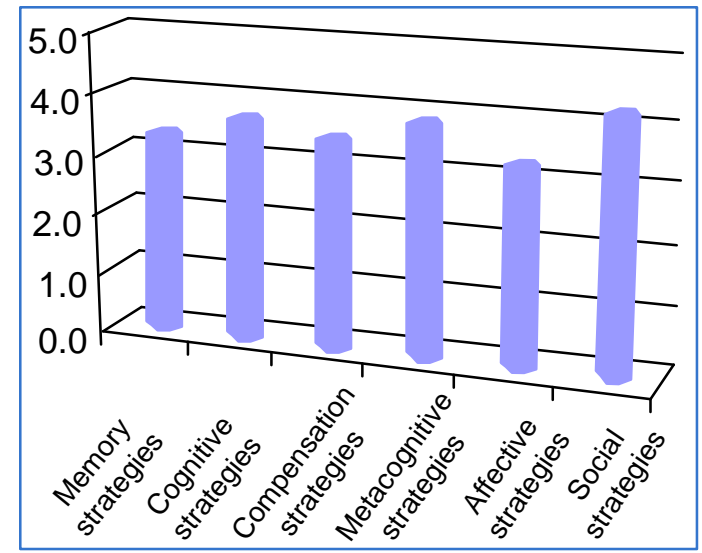

Figure 1: The scores of the six learning strategy groups

The six groups of Chinese language learning strategies used by university students, presented in Figure 1, are as follows: Social strategies, Metacognitive strategies, Cognitive strategies, Compensation strategies, Memory strategies and Affective strategies.

The Social strategies are the most commonly used kind of strategies. According to Profile of Results of Oxford, the Social strategies, Metacognitive strategies and Cognitive strategies are between 3.5 and 4.4, thus the results mean that university students usually use Social strategies, Metacognitive strategies and Cognitive strategies in the process of learning Chinese and the use frequencies are at high level. Except for those three groups of strategies, all of the remaining three strategies fall within the range of 2.5 to 3.4 , thus those figures mean that university students sometimes use these three strategies in the process of learning Chinese and the use frequencies of these three strategies are at medium level. The frequency of the six strategy groups is shown in Table I.

TABLE I Frequency of use for each of the six strategy groups

\begin{tabular}{|c|c|c|c|}
\hline Rank & Strategy name & Frequency & Evaluation \\
\hline 1. & Social strategies & High & Usually used \\
\hline 2. & Metacognitive strategies & High & Usually used \\
\hline 3. & Cognitive strategies & High & Usually used \\
\hline 4 & Compensation strategies & Medium & Sometimes used \\
\hline 5 & Memory strategies & Medium & Sometimes used \\
\hline 5 & Affective strategies & Medium & Sometimes used \\
\hline
\end{tabular}

The results show that the use frequencies of Social strategies, Metacognitive strategies and Cognitive strategies are at high level while the other three strategy groups (those are: Compensation strategies, Memory strategies and Affective strategies) are at medium level.

\section{Discussion}

The term "strategy" in Chinese language consists of two Chinese characters: 策 and 略. In Ancient China, the two Chinese characters were used separately as two words, but both of them had a meaning of "plan" and "attempt to find a solution". For the first time the two Chinese characters were used as one word in the book named History of the Three Kingdoms ${ }^{3}$. "Strategy" means "tactical plan" and "tactical method". Learning strategies can be efficient and effective for students' language learning.

Chinese learning strategies can also be efficient and effective for all of the Chinese language learners. Chinese scores have positive relationship with Chinese language strategies ${ }^{4}$. It is important for students to use learning strategies in the process of the Chinese language learning.

The results show that university students always use Chinese language learning strategies. From high to low order of using the learning strategies, university students use Social strategies, Metacognitive strategies, Cognitive strategies, Compensation strategies, Memory strategies and Affective strategies. They always use the first three strategies and sometimes employ the left three strategies. Those results show that university students have room to elevate the frequency of the Chinese language learning strategies they use, while teachers can further encourage the use of these strategies.

In the process of teaching Chinese as a foreign language, teachers should adopt proper teaching strategies and thus to improve the quality of teaching and to make the teaching more effective ${ }^{5}$. Meantime, as a part of Chinese language teaching, the teachers should teach students to use language learning strategies ${ }^{6}$.

\section{Conclusion}

\section{A. University students always use Chinese language learning} strategies, employing them at high level.

B. The most commonly used by university students kinds of strategies are Social strategies, Metacognitive strategies and Cognitive strategies. University students sometimes use Compensation strategies, Memory strategies and Affective strategies and their use frequencies are at medium level.

\section{References}

[1] Sun Yonghong, A study on Chinese language learning strategies for Bulgarian students, Applied Chinese Language Studies III.UK:London. pp.198-208,2012.

[2] Oxford, Rebecca L., What Every Teacher Should Know, Newbury house, USA: New York, 1990.

[3] Shi Yaofang, Review of research on learning strategy in the twentieth century. Psychological science.5, pp. 586-590, 2001.

[4] Sun Yonghong, A study on relationship between Chinese language learning strategies and Chinese scores, London, in press,2013.

[5] Sun Yonghong \& Xu Xiangdong, On teaching methods and strategies for Chinese language department under non-Chinese circumstances. Modern Education Science. China, vol.5, pp.53-58, 2008. 
[6] Sun Yonghong, How to infiltrate Chinese culture into language teaching of Chinese as a foreign language. "Cultures and Religions of the Balkans, Mediterranean and the Orient”, Bulgaria, pp.431-438, 2011. 